\title{
HOMOLOGY OF GROUP SYSTEMS WITH APPLICATIONS TO LOW-DIMENSIONAL TOPOLOGY
}

\author{
BY S. J. LOMONACO, JR.1
}

Eilenberg-Mac Lane complexes are generalized to GEM complexes. This generalization is then shown to unify many diverse seemingly unrelated concepts in low-dimensional topology. All 2-dimensional $C W$-complexes [1], all 3-dimensional manifolds [5], and all smooth 2-knot exteriors [5] are shown to be GEM complexes. A method is given for computing the (co)homology of the universal cover of a GEM complex from the (co)homology of a naturally associated group system. Hence, this yields a method for computing the second homotopy group $\pi_{2}$ and the $k$-invariant in $H^{3}\left(\pi_{1} ; \pi_{2}\right)$.

\section{GEM complexes.}

Definition. A generalized Eilenberg-Mac Lane (GEM) complex is a $C W$-complex $K$ together with nonempty subcomplexes $K_{-}, K_{0}, K_{+}$such that (1) $K=K_{-} \cup K_{+}$, (2) $K_{0}=K_{-} \cap K_{+}$, (3) each $K_{\lambda}$ is 0 -connected and aspherical (i.e., $\pi_{q} K_{\lambda}=0$ for $q \neq 1$ ) for $\lambda=-, 0,+$. The associated group system $\mathbf{G}=$ $\pi_{1} \mathrm{~K}$ is the collection of groups, $\left\{\pi_{1} K_{-}, \pi_{1} K_{0}, \pi_{1} K_{+}\right\}$together with the morphisms induced by inclusion.

THEOREM 1. Let $K$ and $K^{\prime}$ be two GEM complexes. If an associated group system $\pi_{1} \mathbf{K}$ of $K$ is isomorphic to an associated group system $\pi_{1} K^{\prime}$ of $K^{\prime}$, then $K$ and $K^{\prime}$ are of the same homotopy type. Hence, the name "GEM" and the notation $K=K(\mathrm{G}, 1)$ are justified.

THEOREM 2. For every group system $\mathrm{G}$, the GEM complex $K(\mathrm{G}, 1)$ exists.

REMARK 1. The exterior of every smooth 2 knot $\left(S^{4}, k S^{2}\right)$ is a GEM complex since every 2 -knot has a hyperbolic splitting. (See [5].) (This is a natural 4-dimensional analogue of the asphericity of classical knots [9].) Every 3-manifold is a GEM complex since every such 3-manifold has a Heegaard splitting of positive genus. Every 2-dimensional $C W$-complex has a subdivision which is a GEM complex [1].

II. Group systems.

Definition. Let $\mathbf{G}$ be a group system and let $G$ denote its direct limit

Presented to the Society, August 25, 1979, under the title, Algebraic 3-type and the homology of group systems; received by the editors May 19, 1980.

1980 Mathematics Subject Classification. Primary 55N25, 55N35, 55Q52, 57Q45; Secondary 55S45, 57M05, 57M 10.

1Supported in part by a State University of New York Summer Research Grant. 
(i.e., push-out). (See [3].) Let $C^{\lambda}$ be a free left $Z G_{\lambda}$-resolution of the group $G_{\lambda}$ for $\lambda=-, 0,+$. By a well-known theorem there is a chain map extension $\gamma^{ \pm}: C^{0} \rightarrow C^{ \pm}$of the identity map of $Z$ as a trivial left $Z G_{0}$ module onto $Z$ as a trivial left $Z G_{ \pm}$-module over the morphism $G_{0} \rightarrow G_{ \pm}$. Let $\hat{C}^{ \pm}$denote the left $Z G_{ \pm}$-chain complex which is the mapping cylinder of $\gamma^{ \pm}$and let $i_{ \pm}: C^{0} \rightarrow \hat{C}^{ \pm}$ denote the chain map induced by the inclusion of $Z G_{ \pm} \otimes_{G_{0}} C^{0}$ into $\hat{C}^{ \pm}$. Then the left $Z G$-chain complex which the direct limit (i.e., push-out) of the system $\hat{C}^{-} \stackrel{i}{\longleftarrow} C^{0} \stackrel{i_{+}}{\longrightarrow} \hat{C}^{+}$is called a chain complex of the system $\mathbf{G}$ and denoted by $C \mathbf{G}$.

Proposition 3. Every two chain complexes of a group system $\mathbf{G}$ are chain homotopic.

Definition. Let $\mathbf{G}$ be a group system with direct limit (i.e., push-out) $G$ and let $A$ be a right $Z G$-module. The homology of the group system $\mathbf{G}$ with local coefficients in $A$, written $H_{*}(\mathbf{G} ; A)$, is defined as $H_{*}(\mathbf{G} ; A)=H_{*}\left(A \otimes_{G} C \mathbf{G}\right)$.

III. Main theorem.

THEOREM (MAIN) 4. Let $\mathbf{G}$ be a group system with direct limit (i.e., pushout) $G$. Let $K$ be the GEM complex $K(G, 1)$ and let $\widetilde{K}$ denote the universal cover of $K$. Then every chain complex $C \mathbf{G}$ of $\mathbf{G}$ is chain homotopic to the augmented chain complex $C \widetilde{K}$ of singular chains of $\widetilde{K}$. Hence, $\bar{H}_{*} \widetilde{K}$ is isomorphic to $H_{*}(\mathrm{G} ; Z G)$ as a left $Z G$-module, where $\bar{H}$ denotes the reduced homology.

Corollary 5. By the Hurewicz theorem, the second homotopy group $\pi_{2} \mathrm{~K}$ is isomorphic to $\mathrm{H}_{2}(\mathrm{G} ; Z G)$ as a left $Z G$-module. By the Van Kampen theo$\operatorname{rem}[3], G=\pi_{1} K$.

REMARK 2. The above corollary may be thought of as a $\pi_{2}$-generalization of Crowell's version of the Van Kampen theorem [3]. (See \&V.1). There are other versions of generalized Van Kampen theorems. See for example [2].

COROLlaRY 6. Let $\bar{C}$ be a free left resolution of $G$ agreeing with CG up to and including dimension 2 and having $C G$ as a direct summand. Then the map $\bar{C}_{3} \stackrel{\partial_{3}}{\rightarrow} B_{2}(\bar{C})=Z_{2}(C G) \stackrel{\eta}{\rightarrow} H_{2}(C G)$ is a representative of the $k$-invariant $\mathbf{k} K$ of $K$ lying in $H^{3}\left(\pi_{1} K ; \pi_{2} K\right)$. (See [7].)

Corollary 7. A GEM complex $K(\mathrm{G}, 1)$ is aspherical, i.e., an EilenbergMac Lane space, if and only if $H_{*}(\mathrm{G} ; Z G)=0$.

The following is a generalization to push-outs of Swan's [10] Mayer-Vietoris sequence (with local coefficients) for free products with amalgamation.

Corollary 8. Let $\mathbf{G}$ be a group system with push-out $G$ and let $A$ be a right ZG-module. Then the following is a long exact sequence

$$
\begin{aligned}
\cdots & \rightarrow H_{q+1}(\mathrm{G} ; A) \rightarrow H_{q}\left(G_{0} ; A\right) \\
& \rightarrow H_{q}\left(G_{-} ; A\right) \oplus H_{q}\left(G_{+} ; A\right) \rightarrow H_{q}(\mathrm{G} ; A) \rightarrow \cdots .
\end{aligned}
$$


IV. Methods for computing $H_{*}(\mathbf{G} ; Z G)$. To each presentation (x:r) of a group $G$ there corresponds a Fox-Lyndon resolution where the boundary operators are given by the Jacobians of the Fox derivatives. (See [4, II, p. 210], $[6$, p. 651], and [11, p. 471].) In [5] a method is given for computing a presentation of the left $Z G$-module $H_{*}(\mathbf{G} ; Z G)$ from the Fox-Lyndon resolutions of the groups in G. The kernels of the boundary operators are computed in [5] using the techniques of $[12, \S 8]$. These methods were used in [5] to compute the algebraic 3-type [7] of smooth 2-knot complements and of 3-manifolds.

V. Generalizations. Details for the following will appear elsewhere.

1. The phrases "aspherical structure" and "group system" are used above rather than "aspherical triad" and "group triad" because all definitions and results listed above hold for more general aspherical structures and group systems.

2. All the definitions and results of this paper may be generalized to GEM complexes $K(\mathrm{G}, n)$ of type $n$.

3. The cohomology of the universal cover of GEM complexes and of group systems can also be treated as above.

VI. Sketch of proofs. (1) Theorem 1 is proven by piecing together compatible homotopy equivalences between corresponding sets in the aspherical structures. The existence of such compatible equivalences follows from standard arguments in obstruction theory. The resulting map is shown to be a homotopy equivalence again by standard arguments in obstruction theory. (2) Theorem 2 follows by first applying Milnor's classifying space functor [8] to the group system and then making use of the appropriate reduced mapping cylinders. (3) Main Theorem: Cellular chain complexes $C^{-}, C^{0}, C^{+}$of the universal covers of $K_{-}, K_{0}, K_{+}$are formed by lifting cell decompositions of $K_{-}, K_{0}, K_{+}$. An appropriate chain map $\gamma^{ \pm}: C^{0} \rightarrow C^{ \pm}$over $\pi_{1} K_{0} \rightarrow \pi_{1} K$ is chosen. The push-out is then shown to be exactly the cellular chain complex $C \widetilde{K}$ of the universal cover $\widetilde{K}$ of $K$ obtained by lifting the cell decomposition of $K$ to $\widetilde{K}$.

\section{REFERENCES}

1. William Bechmann, Personal communication.

2. R. Brown and P. J. Higgins, On the connection between the second relative homotopy groups of some related spaces, Proc. London Math. Soc. 36 (1978), 193-212. 43-50.

3. Richard H. Crowell, On the Van Kampen Theorem, Pacific J. Math. 9 (1959),

4. Ralph H. Fox, Free differential calculus. I, Ann. of Math. (2) 57 (1953), 547-560; Free differential calculus. II, Ann. of Math. (2) 59 (1954), 196-210.

5. S. J. Lomonaco, Jr., The homotopy groups of knots. I. How to compute the algebraic 2-type, Pacific J. Math. (to appear).

6. Roger C. Lyndon, Cohomology theory of groups with a single defining relation, Ann. of Math. (2) 52 (1950), 650-665.

7. Saunders Mac Lane and J. H. C. Whitehead, On the 3-type of a complex, Proc. Nat. Acad. Sci. 36 (1950), 41-48. 
8. John Milnor, Construction of universal bundles. II, Ann. of Math. (2) 63 (1956), 430-436.

9. C. D. Papakyriakopoulos, On Dehn's lemma and the asphericity of knots, Ann. of Math. (2) 66 (1957), 1-26.

10. Richard G. Swan, Groups of cohomological dimension one, J. Algebra 12 (1969), $585-601$.

11. H. F. Trotter, Homology of group systems with applications to knot theory, Ann. of Math. (2) 76 (1962), 464-498.

12. J. H. C. Whitehead, On the asphericity of regions in a 3-sphere, Fund. Math. 32 (1939), 149-166.

DEPARTMENT OF COMPUTER SCIENCE AND DEPARTMENT OF MATHEMATICS, STATE UNIVERSITY OF NEW YORK, CENTER AT ALBANY, ALBANY, NEW YORK 12222

DEPARTMENT OF MATHEMATICS AND DEPARTMENT OF COMPUTER SCIENCE, VANCOUVER, BRITISH COLUMBIA, CANADA V6T-1Y4

DEPARTMENT OF MATHEMATICS, UNIVERSITY OF OREGON, EUGENE, OREGON 97403 